\title{
194 EXPERT PERFORMANCE FROM THE PERSPECTIVE OF ERROR IDENTIFICATION
}

Vivekanand Tripathi, Namita Pande Department of Psychology, University of Allahabad, Allahabad, Uttar Pradesh, India

\subsection{6/bjsm.2010.078725.194}

It is a fact that experts perform better than novices. Experts think faster and more accurately than novices. But what happens when a mistake occurs during the performance in a task. How experts and novices perform under such situation, when a mistake has already taken place, and how they find the solutions in an efficient manner? The present study investigates this issue that how experts are better in handling such situation where a mistake has taken place and they intend to rectify it. Error identification, resultant consequences, and rectification abilities, are the few dimensions on which the experts and novices have been assessed. Focused interview of two experts and one novice was done (after blind fold Rubik's cube solving), and the emerging themes were analysed. The present study reveals that while solving Rubik's cube, where a predesigned course of action (pre-programmed steps) was running, when there was any discrepancy found in the real intermediary step and pre-programmed intermediary step, then the experts and novices behave differently. While solving Rubik's cube, experts are able to identify the error earlier than the novices, and in comparison to novices experts handle such situation in a better manner. Experts stated that as soon as they identify an error they immediately start a process of rectification. While novices stated that only when they get stuck with cube, they realise that they did some mistake, and when they tried to rectify it they found it difficult. That makes experts effective, efficient and faster. It does not mean that experts are always correct or error free, they are also prone to mistakes, but most of the times their 'rectification ability' (due to more cumulative experience) helps them to control the situations. So if we include 'error identification and rectification' task in our training programmes than it will positively helps to improve the performance. 